\title{
Changes in alertness and processing capacity in a serial learning task
}

\author{
J. RICHARD JENNINGS, BETSY E. LAWRENCE, and PAUL KASPER \\ Walter Reed Army Institute of Research, Washington, D.C. 20012
}

\begin{abstract}
Second-to-second changes in processing capacity were examined using a concurrent task paradigm involving serial learning and simple reaction time (RT) in two experiments. Experiment 1 demonstrated the feasibility of combining probe RT with a slow-paced serial learning task including perceptually isolated items. Experiment 2 employed the same concurrent tasks to compare cardiac interbeat interval (IBI, the reciprocal of heart rate) with probe RT. Secondto-second changes during item processing were found consistently for both measures. However, these changes appeared to be more related to observing and response requirements than to the specific cognitive processes required by the tasks. Correctly anticipated items were associated with (1) RT changes, suggesting heightened allocation of capacity, and (2) a cardiac IBI response of episodic cardiac deceleration imposed upon the task-induced cardiac speeding. The probe RT and cardiac IBI measures showed reasonable convergence in assessing the allocation of processing capacity. The concept of the accessibility of allocated capacity was introduced in considering instances of the divergence of probe RT and cardiac IBI.
\end{abstract}

Reliable variations of autonomic responses have been observed during a number of information processing tasks (Craik \& Blankstein, 1975; Jennings, 1975; Lacey \& Lacey, 1974). Changes in heart rate, or its reciprocal interbeat interval (IBI), have been of particular interest because the direction of the response differs between different tasks. On the basis of such results, phasic lengthening of the IBI (cardiac deceleration) has been associated with the intensity of attention (Jennings, Averill, Opton, \& Lazarus, 1971; Walter \& Porges, 1976), with the detection and response requirements of an anticipated stimulus (Coles \& Duncan-Johnson, 1975), and with environmental intake (Lacey, Kagan, Lacey, \& Moss, 1963). Phasic shortening of the IBI (cardiac acceleration) has been associated with various cognitive processes, including transient memory load (Jennings, 1971, 1975) and with mental elaboration (Lacey \& Lacey, 1974; Lacey et al., 1963). The diffuseness and variety of these psychophysiological associations may be due largely to the difficulty of providing independent measures of concepts such as attention and mental elaboration. The current investigation attempted to assess cardiac change in a performance task designed to provide an independent measure of the concept under study, processing capacity allocation.

The first author is also affiliated with George Mason University. The second author is now at the University of Pennsylvania and the Institute of Pennsylvania Hospital, and the third author is at the West Haven, Connecticut, Veterans Administration Hospital. Requests for reprints should be sent to J.R. Jennings, Division of Neuropsychiatry, Walter Reed Army Institute of Research, Washington, D.C. 20012. The assistance of John Durkin in collecting the data and the critical reading of Charles $\mathrm{C}$. Wood are gratefully acknowledged.
In the present experiment, cardiac changes during cognitive processing were related to the concept of processing capacity allocation using a concurrent task paradigm. Kahneman (1973) and Kerr (1973) have reviewed the rationale and empirical results relevant to the use of the concurrent task paradigm as a measure of processing capacity. Briefly, performance variations on the less salient of two tasks are used to assess the processing capacity allocated to the more salient of the two tasks. As performance on the less salient of the two tasks decreases, capacity allocated to the more salient task is assumed to increase. Generally, a limited capacity central processor is assumed, which is occupied in either serial or parallel fashion by cognitive operators or contents. The use of secondary task performance as an index of capacity presumes that sufficient capacity is not available to process both tasks optimally and that total capacity is either known or fixed. Kahneman (1973) has suggested that capacity may not be fixed; however, the comparability of secondary task performance across time or events becomes questionable when neither total capacity nor capacity-time devoted to the primary task are known. Kantowitz and Knight (1976) have discussed this as a failure of Norman and Bobrow's (1975) principle of complementarity. Under appropriate conditions, however, secondary task performance may provide a sensitive index of capacity that can be used to probe the precise timing of information processing requirements (e.g., Becker, 1976). Norman and Bobrow (1975, 1976; see also Kantowitz \& Knight, 1976) have provided a general discussion of concurrent task paradigms suggesting their utility for the study of performance limitations.

Two experiments are reported using a serial learning paradigm designed to allow joint assessment of 
secondary task performance and changes in cardiac IBI. The first experiment assessed the feasibility of using the concurrent task paradigm in a slow-paced task suitable for measurement of IBI. The second experiment obtained measures of IBI as well as subjective ratings of mental effort. In both experiments, stimuli for the secondary task, simple RT, were presented at different points during item presentation in order to assess any second-to-second changes in the allocation of processing capacity. The initial experiment assessed the sensitivity of the concurrent task technique by varying the types of items to be learned, numbers vs. words, and by including perceptually isolated von Restorff items (see Wallace, 1965). Both manipulations were thought to influence the allocation of processing capacity.

\section{EXPERIMENT 1}

\section{Method}

Subjects. Twenty young adult volunteers were paid to serve as subjects. Each subject participated on 2 consecutive days. On 1 day they received lists of numbers and on the other, lists of words to memorize. Half of the subjects received reaction time probes and half did not. Order of words and numbers and probe vs. no-probe conditions was randomly determined.

Experimental task. The experimental task consisted of serial anticipation of 12 lists of either 13 two-digit numbers or 13 high-frequency words (double A nouns, Thorndike \& Lorge, 1944). Items were presented once every $4.5 \mathrm{sec}$, using slides rear-projected onto a screen positioned on a table directly in front of the subject. Fach list was presented for a single trial composed of two list presentations. Subjects were asked not to verbalize responses during the initial or "learning" presentation. The second presentation followed immediately, commencing with a blue slide containing a white dot in the center to signal anticipation of the first item in the list. Anticipation was paced by the appearance of each successive item (i.e., method of serial anticipation). Feedback regarding number of correct items was given after every two lists. Successive lists were separated by three blank slides.

One-half of the lists contained one or two perceptually isolated words that appeared randomly in Positions 4-10, with the constraint that these items not appear consecutively. Regular words or numbers were displayed on a blue background. Perceptually isolated items were displayed on a red background.

Subjects were seated in a sound-attenuating chamber. White noise at approximately $15 \mathrm{db}$ SL was presented binaurally over earphones throughout each session. For half of the subjects, reaction time (RT) probes (a 1,000-Hz, 100-msec, 40-db SL tone) were presented, requiring the subject to respond as quickly as possible by pressing a microswitch key placed on the arm of the chair corresponding to his preferred hand. During half of the items, no tone was presented; for the other items, the tone occurred with equal probability after $0-, 5-, 1.5-, 2.5-$, or $3.5-\mathrm{sec}$ delays from word onset. Two "lists" containing 64 blank slides with random RT probes were presented at the beginning and end of each session. Stimulus presentation and timing were accomplished with a logic system composed of BRS and Coulbourn Instruments modules. The stimulus number, probe length, and RT on each trial were recorded with a Hewlett Packard 5050B printer. A Coulbourn voice-key module was used to code the time of occurrence of the verbal anticipations relative to the occurrence of probe RT stimuli.

\section{Results and Discussion}

The results for serial anticipation showed the expected serial position effects and relative difficulty of numbers over words. Isolated words were anticipated more correctly than comparable nonisolated words (Wilcoxin $\mathrm{T}=51.5, \mathrm{n}=20$ ). A significant isolation effect was not obtained for numbers $(T=113.5)$.

Serial anticipation for the group with the concurrent probe RT task did not differ from the unprobed group. Mean proportion recalled was .41 for the probed group and .42 for the unprobed group. This result suggests that the concurrent probe RT task caused little if any interference with the primary serial anticipation task.

Figure 1 presents the mean probe RTs at the five probe times separately for learning and anticipation phases of the task. The learning phase results are presented separately for isolated and nonisolated items. The mean data presented are the averages of median RTs computed for each individual's data. Reaction times for responses occurring after overt verbal anticipation were not included in the anticipation data.

As can be seen in Figure 1, RT during anticipation was slower than during learning. The mean RT during the presentation of "blank" items was $290.4 \mathrm{msec}$, substantially faster than that for either learning or anticipation. These findings are in agreement with previous findings of concurrent task studies of memory performance (see Kerr, 1973). The differences between the means for blank, learning, and anticipation were all significant by post hoc Tukey b tests (Winer, 1971) performed after finding a significant effect of phase $[\mathrm{F}(\operatorname{adj} 1,16)=35.67]$ in an analysis of variance that also included probe time, order group, and words vs. numbers as factors. A .05 rejection level was used and degrees of freedom were adjusted to account for nonhomogeneity of covariances (see Jennings \& Wood, 1976).

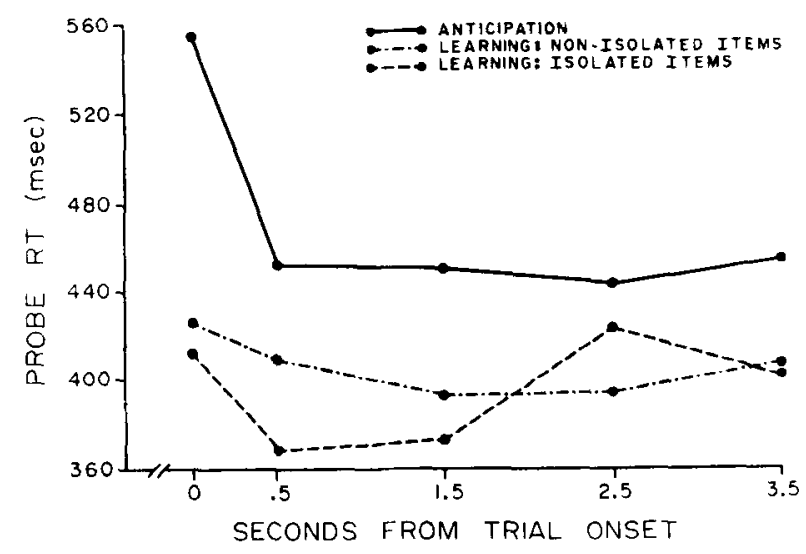

Figure 1. Mean probe RT at the five probe times presented separately for the anticipation and learning portions of the trials. The learning results are further subdivided for nonisolated and isolated items. 
The differences between the different probe times in Figure 1 show that probe RTs were slowed maximally when the probe signal was coincident with the onset of a learning or recall item [Phase by Probe Time: $F($ adj 1,64$)=4.93$ ]. Post hoc tests showed for the learning probe times that the $0-\mathrm{sec}$ delay (coincident with item onset) probe RT was different from those for the 1.5- and $2.5 \mathrm{sec}$ delays. For the anticipation probe time results, the $0-\mathrm{sec}$ delay mean was different from all the means for the remaining probe times. On the basis of these results, cognitive processes occurring soon after item onset appear to require greater allocation of processing capacity than processes occurring later in time.

Opportunities to probe isolated items were limited by the design; only 8 to 14 probe RTs were collected at each probe time. As shown in Figure 1, these RTs appeared different from RTs during nonisolated items. The paucity of data points preclude a statistical analysis; however, the size of these effects may be compared to the variability of nonisolated probe RTs in Serial Positions 4-10. For these data the average standard deviation was 62.9 (range over probe times 45.3 to 77.2) and the standard error of the mean based on this was 19.9. Although differences at two of the probe times are slightly beyond confidence limits using the standard error of the mean, this statistic is not strictly appropriate. The initial speeding and subsequent slowing of RT during isolated relative to nonisolated items, though suggestive, must be considered tentative prior to replication of this result.

The logic of the concurrent task paradigm suggests that because isolated items were anticipated more correctly, they should be associated with slower probe RTs than the nonisolated items. This, however, did not occur. An alternative related to the viewpoint of Kahneman (1973) might be advanced. The isolated items, due to their perceptual disparity from the remaining items, are likely to induce a brief increase in overall processing capacity, allowing a secondary task to be performed efficiently with less interference with the primary task. This view must assume that the additional capacity produced through the perceptually induced orienting process is transient and not strictly focused on the primary (serial anticipation) task. An interpretation maintaining the fixed capacity assumption might suggest that the perceptually isolated items initially direct task-allocated capacity to the visual display of the item. This allocation of capacity to observation of the environment may facilitate responses to a probe RT stimulus.

The relation of correctness of anticipation to probe RT was examined indirectly by comparing RTs from Serial Positions 1-4 (mean proportion correct, .69) with Positions 7-10 (proportion correct, .25). Table 1 presents the mean probe RTs by probe delay time. The mean for RTs to the blank slides are included for comparison. An analysis with factors for probe time, serial position, and phase (learning vs. anticipation) showed that the only significant effect of serial position was an interaction with phase $[F(1,9)=12.96]$. As seen in the last column of Table 1 , serial position influenced RTs during anticipation but not learning. Relatively correct anticipations were associated with larger allocations of processing capacity than incorrect or omitted anticipations.

\section{EXPERIMENT 2}

Experiment 1 suggested that processing capacity and its variation could be reasonably assessed in a slowpaced serial learning situation. This result made it possible to study changes in IBI in a cognitive task in which an independent measure of processing capacity allocation could be obtained. In the context of the discussion of Experiment 1, IBI may be of use as an independent measure of perceptually induced changes in the amount of temporal allocation of processing capacity (e.g., Lacey et al., 1963).

As well as including IBI, Experiment 2 varied degree of learning rather than item difficulty and attempted to assess momentary effort. In Experiment 1 a practice effect had confounded the assessment of item difficulty. Therefore, in Experiment 2, subjects were practiced thoroughly and degree of learning was used to manipulate processing requirements. If serial lists are presented over trials, items that were previously learned shouid require less processing capacity on subsequent learning and recall trials. Thus, the probe RT should reflect increased available processing capacity on successive trials of an initially correct item (cf. LaBerge, 1975; Norman \& Bobrow, 1975). Subjective ratings of momentary effort were collected to relate to IBI and probe RT empirically and to relate interpretively to Kahneman's (1973) theoretical concept of effort.

\section{Method}

Subjects. Twenty-four college-aged volunteers 10 females and 14 males, mear age, 22 years) were randomly split into two experimental groups. One group of 12 , the probe group, received a dual task consisting of serial learning and a simple

Table 1

Probe Reaction Time in Milliseconds

\begin{tabular}{ccccccc} 
Serial & \multicolumn{6}{c}{ Probe Delay } \\
\cline { 2 - 6 } Position & 0 & .15 & 1.5 & 2.0 & 2.5 & Mean \\
\hline \multicolumn{7}{c}{ Learning } \\
$1-4$ & 430 & 393 & 381 & 390 & 404 & 400 \\
$7-10$ & 409 & 414 & 393 & 397 & 420 & 407 \\
$7-4$ & 564 & 525 & 517 & 485 & 489 & 516 \\
$7-10$ & 577 & 460 & 421 & 445 & 471 & 475 \\
& \multicolumn{7}{c}{ Anticipation } \\
Combined Slides & 291 & 280 & 288 & 291 & 297 & 289 \\
\hline
\end{tabular}


RT task. The other, no-probe, group received only the serial learning task. Interbeat interval responses were collected for both groups but analyzed only for the no-probe group. All subjects received a training session on an initial day and then two experimental sessions on 2 subsequent days within a 10-day period.

Experimental task. A number of the features of the task used in Experiment 1 were changed. Subjects received three successive trials on each word list rather than one trial. As before, each trial was composed of a learning phase, during which subjects observed the serial presentation of the list without verbal responding, and an anticipation phase, during which subjects attempted to anticipate successive items verbally. Thus, this was a modified serial anticipation task. On each day subjects received three trials on each of six lists composed of 11 items each. A different set of six lists was used on each of the 3 days with the order of the lists randomized across subjects. Thorndike-Lorge (1944) double A nouns were selected randomly to form the lists. Time between items was reduced to $4 \mathrm{sec}$.

The probe group received probes at four rather than five probe times relative to slide onset: $0, .35,1.35$, and $2.35 \mathrm{sec}$. As previously, probes were presented randomly on half of the items. During serial anticipation, however, a voice switch was used to lock out probes during the subject's verbalization of his response. The six lists on each day were preceded and followed by a control or rote list also presented for three trials. This list consisted of the numbers from 11 to 21 in arithmetic order. The subjects were requested to perform with this list exactly as they did for the experimental lists, including verbal responses during the anticipation portion of the trial.

Procedure. At the beginning of each session, all subjects had electrodes attached for the measurement of heart rate and respiration. Heart rate electrodes (Lexington Instruments) were filled with conductive jelly (Lexington Instruments) and attached to the sternum, lateral margin of the chest, and ankle. Respiration was recorded using a thermistor placed in the nose using a lightweight earring. Signals were conditioned using a Beckman Type RM dynograph and recorded on a Tandberg FM tape recorder. Interbeat intervals were subsequently computer averaged to form second-to-second mean IBIs (see Jennings, 1975). Averaging began at the beginning of each interval and was terminated at the end of Second 4.

Information on the average number of correct verbal responses and, where appropriate, speed of RT was given after each list. Subjects were rewarded with a $\$ 1.50$ bonus if the proportion of correct anticipations $[\mathrm{P}(\mathrm{C})]$ was over .85 for the session and were also rewarded 1 cent for every RT shorter than $300 \mathrm{msec}$.

Following the final day's performance, a questionnaire was administered that asked for estimations of the amount of effort required at different time points within the task. Subjects were asked to consider 10 as the most effort expended on anything in their life, and then to rate the current task from 0 to 10 in each of the four 1-sec periods following item presentation in learning and anticipation. Subjective reports of strategies used to perform the task were also collected.

Parameters of the probe stimulus and other details of the task and procedure were identical to those in the initial experiment.

\section{Results and Discussion}

Results will be presented in four sections: (1) the results for the primary task, proportion of correct serial anticipations; (2) general analyses of probe RT, IBI, and effort; (3) changes over trials, and (4) analysis of RT and IBI for correct and incorrect serial anticipations. Isolated or von Restorff items were presented; however, these items did not influence serial

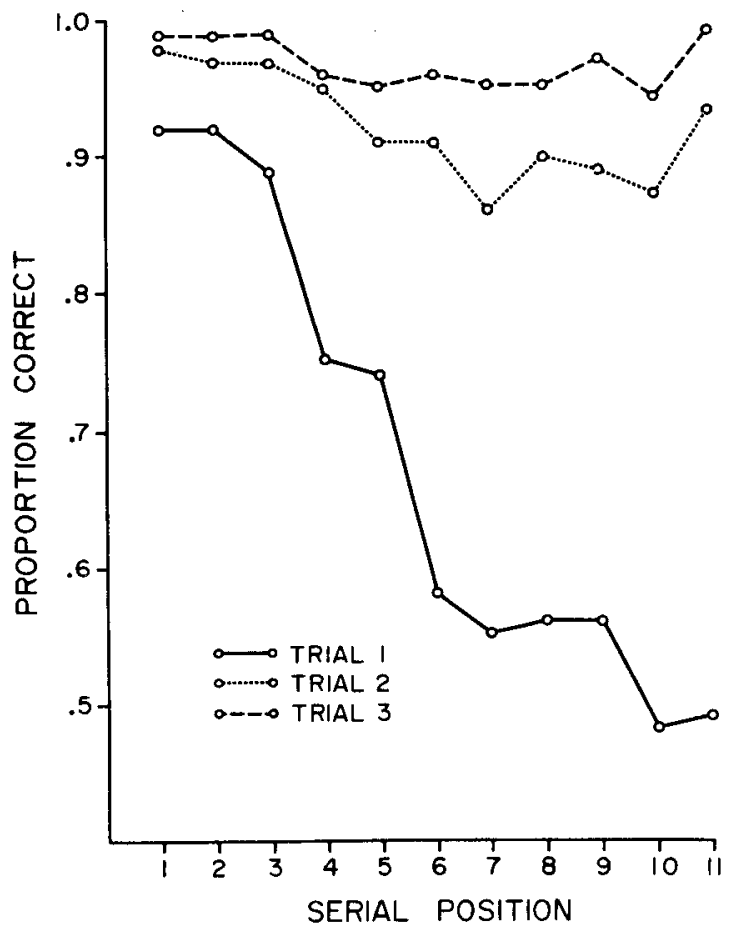

Figure 2. Proportion of anticipations correct as a function of serial position of the item. The results are presented separately for each trial.

anticipation. In the absence of an effect on serial anticipation, isolated items could not be judged perceptually salient. Therefore, data from these trials are not reported. ${ }^{1}$ Data were merged from the 2 experimental days for probe RT and IBI.

Serial anticipation. Figure 2 shows the progressive and expected increase in proportion correct over the three trials of the experiment. Essentially perfect performance was achieved on the initial serial positions and performance increased progressively over trials at the later serial positions.

Serial learning was largely uninfluenced by the probe RT task. The mean proportion of correct serial anticipation for the no-probe group was .86 and the mean for the probe group was .85 . Similarly, performance for the 2 experimental days was essentially the same: Mean $\mathrm{P}(\mathrm{C})$ for Day 1 was .85 and for Day 2 , 86 .

General analysis. The convergence of probe RT, IBI, and effort ratings was examined between conditions that might be expected to show different allocations of processing capacity. ${ }^{2}$ First, RT and IBI may be compared between rote and experimental lists. Second, RT, IBI, and rated effort may be compared between learning and anticipation phases. Third, RT, IBI, and effort may be compared over the seconds during item presentation. Finally, IBI and RT may be compared over the three trials given for each list.

Figures 3 and 4 provide the means necessary to make all the above comparisons for probe RT and cardiac 


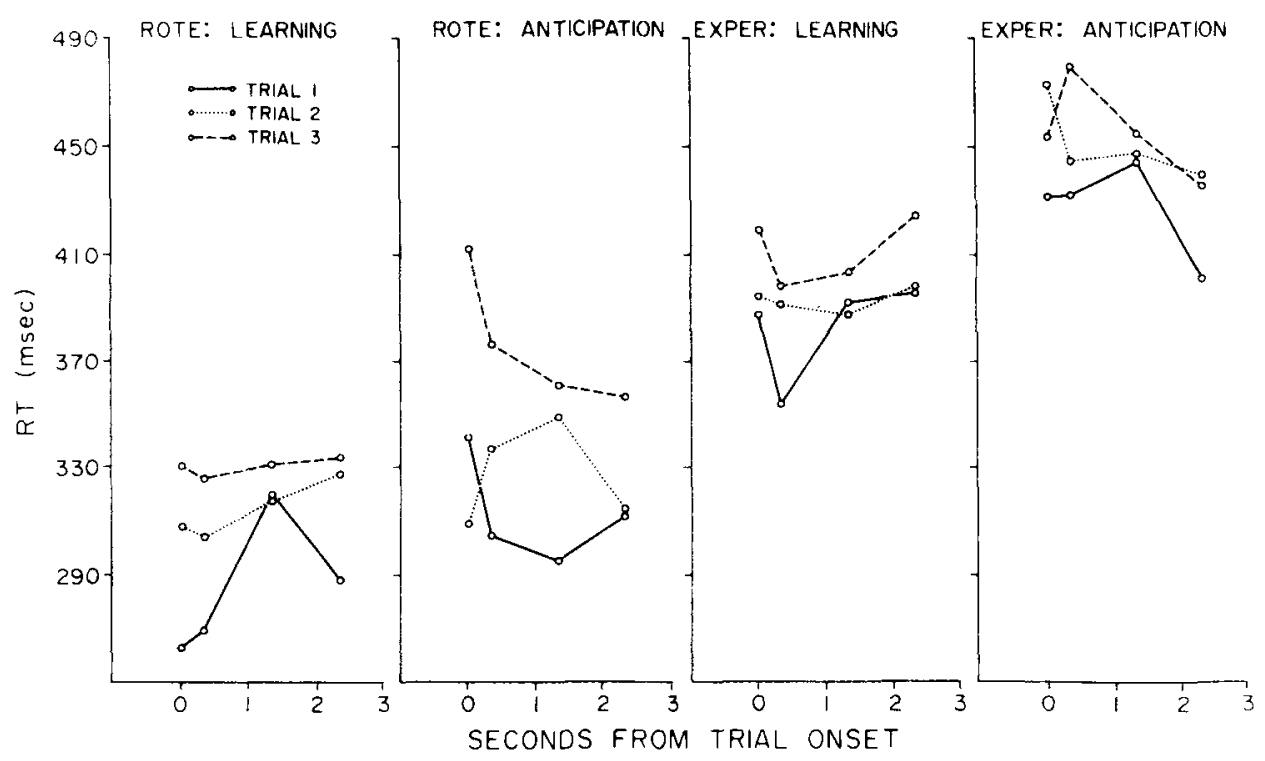

Figure 3. Mean probe RT at each probe time presented over trials for the learning and anticipation phases of the rote and experimental tasks. The rote task consisted of the presentation and anticipation of the sequential numbers from 11 to 21 .

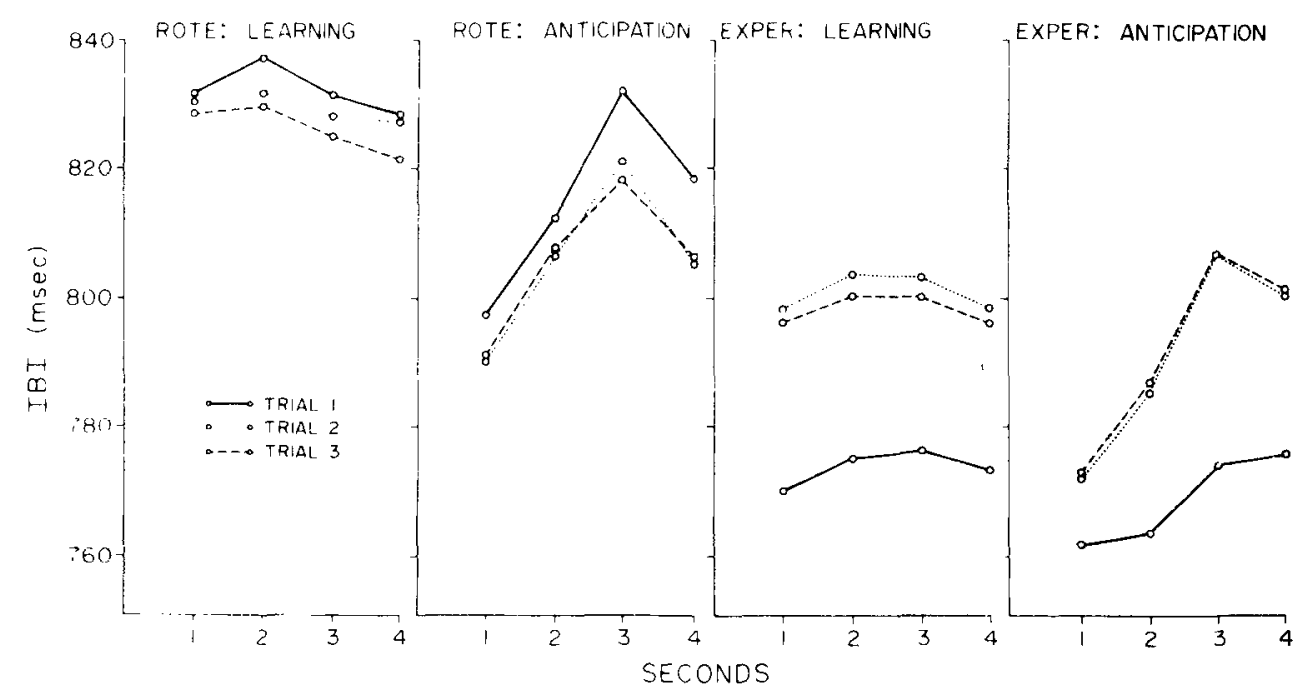

Figure 4. Mean IBI over seconds for the three trials of the experiments. Interbeat in terval was averaged at the end of each second. The separate panels present the results for rote "learning and anticipation" and experimental learning and anticipation.

IBI. Both figures present data classified by task (experimental or rote), phase (learning or anticipation), trials, and second during item presentation. Figure 3 shows the progressive slowing of probe RT from rote learning through rote anticipation and experimental learning to experimental anticipation. Figure 4 shows the progressive shortening of mean IBI as the same comparison is made. Subsequent sections will examine the apparent inverse relation of IBI and RT over most of the variables of interest.

Interbeat interval and RT were analyzed with a fourway repeated-measures analysis of variance with factors for task, phase, probe times or seconds, and trials
(Winer, 1971). Significant effects from this analysis will be discussed factor by factor.

Task and phase results. The probe RT results for task and phase replicated the results of Experiment 1. Probe RT was higher during the experimental task (learning and anticipation of words) (mean $=432 \mathrm{msec}$ ) than during the rote task ("learning and anticipation" of the ordered numbers $11-21$ ) (mean $=343 \mathrm{msec}$ ); and the serial anticipation phase produced longer RTs (mean $=409 \mathrm{msec}$ ) than the learning phase $($ mean $=367 \mathrm{msec}) \quad$ [task: $\mathrm{F}(1,11)=14.38 ;$ phase: $\mathrm{F}(1,11)=7.40]$.

Decreases in IBI paralleled the increases in probe 
Table 2

Effort Ratings

\begin{tabular}{lccccc}
\hline & \multicolumn{5}{c}{ Seconds } \\
\cline { 2 - 6 } & 1 & 2 & 3 & 4 & Mean \\
\hline Learning & 8.0 & 7.5 & 6.2 & 5.8 & 6.8 \\
Anticipation & 7.5 & 6.0 & 4.3 & 4.3 & 5.6 \\
Mean & 7.8 & 6.8 & 5.3 & 5.1 & \\
\hline
\end{tabular}

RT. As seen in Figure 4, mean IBI was shorter in the experimental task than in the rote task. A similar decrease is seen between the learning and anticipation phases of the tasks. [Corresponding $F$ values were for task, $F(\operatorname{adj} 1,11)=14.61$; and for phase, $F($ adj 1,11$)=8.37$.] A significant Task by Phase interaction $[F(\operatorname{adj} 1,11)=5.64]$ reflected the relatively smaller difference between learning and anticipation phases in the experimental task. The same relative differences can be seen in Figure 3 for probe RT, however, as noted above, the appropriate interaction was not significant for this variable. Overall, the task and phase results show a clear convergence of probe $\mathrm{RT}$ and cardiac IBI results.

Table 2 presents the means of the effort ratings which were collected at the end of the experiment. The data are cross-classified by second and phase. The last column shows that the learning phase was rated as requiring more effort than the anticipation phase. Results of a Friedman nonparametric analysis of variance (Mosteller \& Rourke, 1973) supported this difference $\left[\chi^{2}(3)=25.2\right]$. A preliminary parametric analysis of variance had indicated that phase and seconds did not interact. Note that if processing capacity allocation is associated with greater effort, then these results diverge from those for probe RT and IBI.

Second-to-second results. A convergence between probe RT and cardiac IBI for second-to-second changes can be seen by comparing Figures 3 and 4 . Variability in the RT results somewhat obscures this convergence. Both IBI and RT showed relatively small second-tosecond changes during learning. Changes that did occur maintained the inverse relationship between IBI and RT (e.g., at Second 3 of the rote learning, RT is relatively long and IBI relatively short). During anticipation, RT and IBI changed relatively clearly over time. Again RT decreased over seconds and IBI increased. For IBI, the changes over seconds are supported by significant $F s$ for seconds $[F($ adj 1,11$)=8.65]$, Task by Second $[F(\operatorname{adj} 1,11)=4.06]$, and Phase by Second $[F(\operatorname{adj} 1,11)=9.82]$. For probe RT, the changes over seconds were reflected in a significant Probe Time by Phase interaction $[\mathrm{F}(\operatorname{adj} 2,25)=4.35$ ]

A second issue for probe RT is whether the results for Experiment 2 replicate those in Experiment 1. Figure 5 plots mean probe RT for the Probe Time by Phase interaction to facilitate this comparison. The general features of the results clearly replicate: (1) During anticipation, probe RTs are slower at the beginning than at the end of item presentation, and (2) during learning, probe RTs relative to item onset are initially fast and then slower. The results are not precisely the same, however. In Experiment 2 the results for anticipation ( 1 above) were supported by post hoc analyses of the means, but the results for learning (2 above) showed no significant differences between the means. Thus, the significant slowing of probe RT at learning item onset found in Experiment 1 was not replicated in Experiment 2.

Second-to-second changes did not differ markedly between the rote and experimental tasks. The addition of a meaningful task did not change the relative values over time of either IBI or probe RT. The general similarity between rote and experimental results can be seen in Figures 3 and 4. Probe Time (or Second) by Task by Phase interactions were not significant for either RT $[F(3,33)=2.47]$ or $\operatorname{IBI}[F(\operatorname{adj} 1,15)=2.95]$.

Effort ratings decreased over seconds in both learning and anticipation. Table 2 presents this data. The decline over seconds was tested with a Friedman nonparametric analysis of variance [resulting in $\chi^{2}(3)=25.2$ ] (Mosteller \& Rourke, 1973). The clear decline in effort during learning items was inconsistent with the absence of such a change in either RT or IBI. Correlations computed between effort ratings and RT and IBI supported the divergence of these measures. No correlations were significant between second-to-second effort and RT. Interbeat interval was positively related to effort ratings during the $3 \mathrm{rd} \mathrm{sec}$ of learning items $(r=.66)$ and the $1 \mathrm{st} \mathrm{sec}$ of anticipation $(r=.61)$. This means, however, that longer IBIs were associated with greater rated effort. This is the inverse of the relationship expected: greater capacity allocation

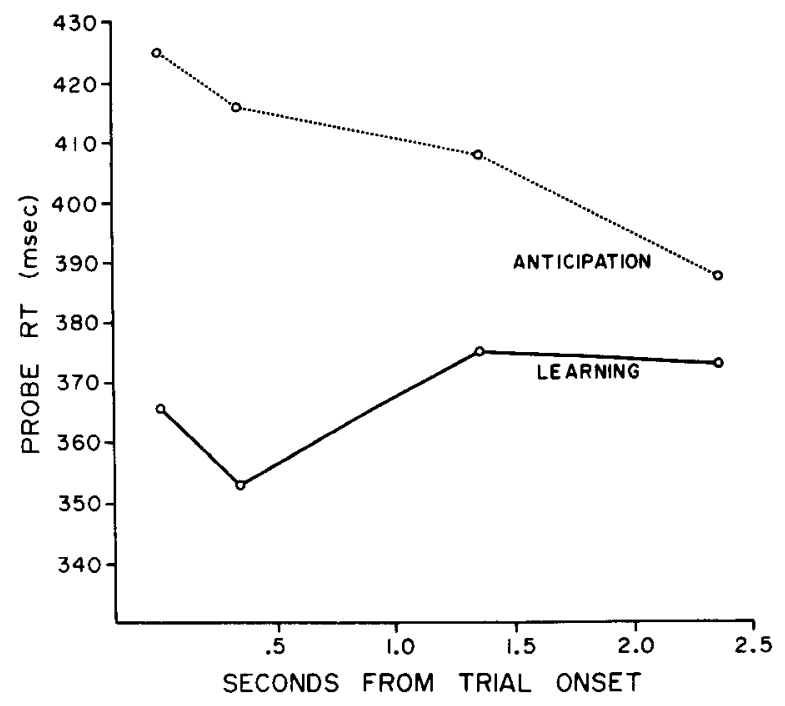

Figure 5. Mean probe RT at the four probe times presented separately for learning and anticipation. 
presumably leading to greater effort and shortened IBIs.

Changes over trials. Over trials probe RT was expected to decrease and IBI increase as items in the experimental task were mastered and more capacity became available. As seen in Figure 4, this occurred for IBI during the experimental task. As might be expected, the rote task was associated with a change in the opposite direction, that is, IBI decreased over trials [trials: $F(\operatorname{adj} 2,20)=3.79$; Task by Trial: $F(\operatorname{adj} 1,11)=5.69$; Second by Trials by Phase by Task: $F(\operatorname{adj} 1,11)=5.99]$. As seen in Figure 3, the probe RT results for trials diverged from those for IBI. Reaction time increased rather than decreased over trials $[F(\operatorname{adj} 1,11)=18.29]$. Additional analyses were done to explore this increase in RT over trials.

The first analysis of RT examined trial effects on items that were correctly anticipated on all three trials (i.e., items that should have been well learned by Trial 3]. This analysis, which had trials and phase as factors, failed to show a significant trials effect $[F(2,22)=2.2]$. The trial means were: Trial $1=428.0$, Trial $2=434.5$, and Trial $3=443.8$. Thus the trend followed that in the data taken as a whole, but the size of the differences between trials was substantially smaller. Comparable examination of IBI data revealed very little difference from the overall results seen in Figure 4.

A second analysis of probe RT examined the possibility that capacity devoted to the tasks declined over an experimental session or, more colloquially, that the subjects reduced their involvement in the tasks. This analysis took data from the initial rote "list" and the initial two experimental lists. If a drop in involvement occurred, the results of this analysis should differ from those of the general analysis. This was the case. A significant trials main effect $[F(\operatorname{adj} 1,11)=10.02]$ reflected means that decreased from $400.2 \mathrm{msec}$ on Trial 1 to $368.5 \mathrm{msec}$ on Trial 2, and then rose slightly on Trial 3 to $374.7 \mathrm{msec}$. This effect was seen more clearly during the experimental task and during anticipation; however, the appropriate interactions did not reach significance.

The results of these additional analyses suggest that the increase in RT over trials seen in the general analyses was due primarily to RTs from the latter part of the experimental session and, secondarily, to relatively long RTs to items that were incorrectly anticipated on Trials 2 and 3.

Correctness analyses. The influence of correctness of anticipation was assessed for both RT and IBI, although the analysis of RT was limited. Probe RTs occurred on only the one-half of the items and the number of incorrect anticipations on Trials 2 and 3 was small. The available data permitted only a two-way analysis of phase and correctness with the RT data for Trial 1 collapsed across probe times. Interbeat interval was analyzed with a repeated-measures design similar to the general analysis, but with a correctness factor replacing the task factor. In these analyses an item was considered correct during learning if it was subsequently anticipated correctly.

Both RT and IBI were sensitive to item correctness, but RT and IBI were both longer for correct relative to incorrect iterns. The inverse relation seen in previous analyses was not found.

Mean correct item probe RT was $454 \mathrm{msec}$ and mean incorrect was $413 \mathrm{msec}[\mathrm{F}(1,11)=5.0]$. For probe $\mathrm{RT}$, correctness did not interact significantly with phase. The relative slowness of probe RT to correct items replicates the findings of Experiment 1 that probes of Serial Positions 1-4 produced slower RTs than those of Positions 7-10. In Experiment 1, however, a significant interaction suggested that this effect was primarily from the anticipation phase. The means from Experiment 2 show only a slight trend in this direction (learning: $\quad$ correct $=450 \mathrm{msec}$, incorrect $=416 \mathrm{msec}$; anticipation: correct $=457 \mathrm{msec}$, incorrect $=409 \mathrm{msec}$ ). In Experiment 2 probe RT was analyzed separately for serial position. In this analysis serial position was partially confounded with correctness; however, the resulting $F$ s for serial position were less than those for the correctness analysis. Interbeat interval from Experiment 2 could be analyzed for serial position, holding correctness constant. In this analysis serial position failed to show any significant main effects or interactions. These results suggest that serial position may have little direct effect on processing capacity when performance on the items is held constant (see Kerr, 1973).

The average IBI response for items that were correctly and incorrectly anticipated are presented separately in Figure 6. The learning phase results in

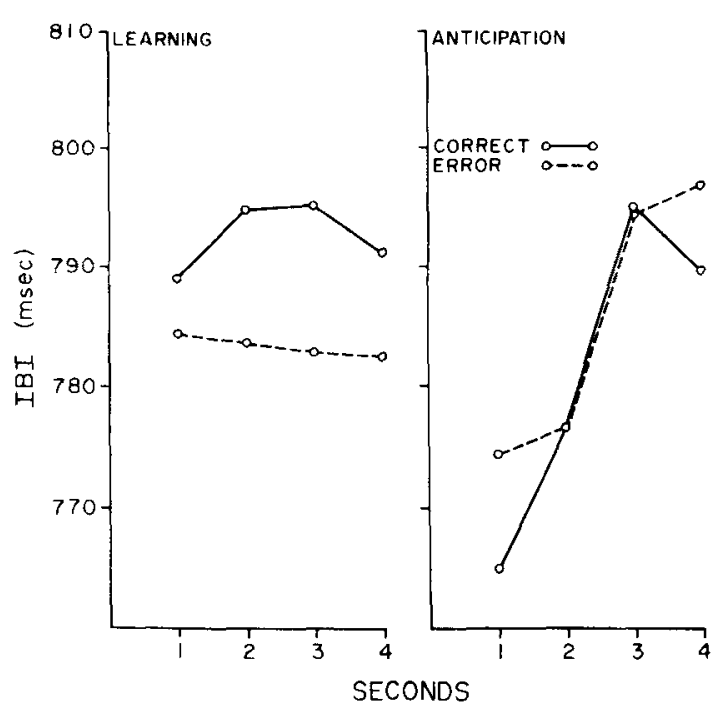

Figure 6. Mean IBI over seconds for items anticipated correctly and incorrectly. The two panels present the results for learning and anticipation. 
the left panel show that items subsequently anticipated correctly were associated with relatively longer IBIs, particularly at Seconds 2 and 3, compared to the IBIs of items subsequently anticipated incorrectly. The right panel of Figure 6 shows the IBI response during anticipation of correct and incorrect items. At Seconds 1 and 4 of anticipation, correct items show shorter IBIs than incorrect items.

As might be anticipated from Figure 6, the IBI results for Phase by Correctness [F(adj 1,11) $=18.11$ ] and for Correctness by Seconds [F(adj 1,11) $=6.77$ ] were significant. A Correctness by Trials by Phase $[F(\operatorname{adj} 2,20)=3.59]$ interaction also occurred, reflecting an accentuation of the differences portrayed in Figure 6 for Trial 1 relative to Trials 2 and $3 .^{3}$

\section{GENERAL DISCUSSION}

\section{Assessment of Processing Capacity}

The major purpose of the current investigation was to compare IBI changes during an information processing task to a relatively well accepted measure of the allocation of processing capacity, that is, performance on a secondary probe RT task. The influence of a large difference in allocated processing capacity on IBI and RT was assessed by comparing items requiring learning to items requiring rote or no responses. Overall probe RT was consistently increased and IBI was shortened for items requiring learning. A second comparison of presumed differences in allocated processing capacity was between the learning and anticipation phases of the task. Previous investigations of secondary task performance during learning and recall (e.g., Johnston, Wagstaff, \& Griffith, 1972; Trumbo \& Milone, 1971) found, as the current study did, that the recall of learned items produced larger decrements in secondary task performance than the learning of the items. As in the comparison of rote and experimental items, increases in RT were paralleled by decreases in IBI (i.e., cardiac IBI during serial anticipation was shorter than during learning). Similar support for an inverse relation between probe RT and cardiac IBI was seen in second-to-second changes during item presentation.

The association of lengthened probe RT and shortened IBI suggests that both may be related to allocation of processing capacity. Thus, assuming a fixed total processing capacity, probe RT should increase and IBI decrease as a larger amount of the total capacity is allocated to a task. As noted in the Introduction, decreases in IBI (cardiac acceleration) have been observed in a variety of cognitive tasks and do not seem to be uniquely associated with a specific cognitive function, such as digit transformation or memory scanning (see, e.g., Jennings, 1975; also, reviews by Hahn, 1973; Kahneman, 1973; Lacey \& Lacey, 1974; Pribram \& McGuiness, 1975). Thus, previous findings are consistent with the present interpretation, while the current results associating an independent, concurrent task measure, probe RT, with cardiac acceleration provide a strong empirical basis for the interpretation of cardiac acceleration as a correlate of the allocation of processing capacity.

Cardiac deceleration, as well as acceleration, has, however, been frequently observed in cognitive tasks, and was observed in the present second-to-second IBI results. Deceleration has been most clearly associated with the anticipation or careful observation of events (Hahn, 1973; Jennings \& Wood, 1977; Lacey \& Lacey, 1974). We propose that the direction of IBI change during a processing task (relative to average IBI or a pretask baseline) is related to the accessibility of processing capacity. Lengthening of IBI indicates that capacity is held available for expected input. Shortening of IBI indicates that capacity is unavailable; that is, processing is ongoing. Degree of change of IBI reflects the allocation of capacity, for example, the amount of capacity accessible or the amount of capacity inaccessible. Note that the nature of the ongoing operations in the processor are not thought to influence IBI; access not content is the primary variable.

The similarity of the interpretation to the early formulation of the Laceys (e.g., Lacey et al., 1963) should be acknowledged. In their fomulation, cardiac deceleration was related to the acceptance of sensory input and cardiac acceleration to the rejection of input. Our interpretation hopefully provides a clear, testable expression of the concept of Lacey and Lacey (1974). We will use the term "alertness" as a brief expression of our concept of the holding available of capacity (cf. Posner, 1974).

\section{Second-to-Second Changes in Allocation}

A second purpose of these experiments was to provide a fine-grained assessment of changes in the allocation of processing capacity by measuring IBI and probe RT on a second-to-second basis. Second-tosecond changes in allocation would suggest that the cognitive operations characterizing different information processing stages require varying amounts of capacity (Kantowitz \& Knight, 1974). Examining the results exclusively in terms of the relative differences between seconds showed first that the results for rote repetition paralleled the results for the experimental task. The presence of a meaningful task did not change the secondto-second results. Second, these analyses showed that anticipation consistently produced results suggesting a decline in allocated processing capacity over seconds, probably induced by the sequence of responding and anticipating required by both the rote and experimental tasks. Finally, changes across seconds in learning were small and for probe RT inconsistent between experiments. Kahneman (1973) has reviewed evidence suggesting that capacity allocation is strongly influenced 
by response related requirements. Changes in probe RT and IBI over seconds seem to reflect such an influence in the current results. Thus, changes in allocation of capacity over seconds seemed less related to detailed cognitive processes and more related to more gross changes in the accessibility of processing capacity as the activity required by the task shifted between responding and observing. The IBI results for anticipation (Figure 4) are a particularly clear example of this shift over time in accessibility.

An attempt was made to relate subjective ratings of effort for each second to probe RT and cardiac IBI. Rated effort was expected to vary directly with processing capacity allocated (Kahneman, 1973). In contrast to the convergence between RT and IBI, however, effort ratings over seconds and between learning and anticipations were not consistent with the other measures. Subjective ratings of effort, particularly the current postexperimental ratings, may be based on a complex of influences unrelated to IBI and probe RT and the concept of Kahneman (1973).

\section{Performance Correlates}

Probe RT and IBI showed significant relations to the proportion of items correctly anticipated. The slowing of probe RT for correct as compared to incorrect items suggested that items processed with a larger allocation of processing capacity were anticipated correctly (cf. Kahneman, Beatty, \& Pollack, 1967).

The primary correct vs. error differences in IBI occurred during item learning. On items subsequently anticipated correctly, a brief relative deceleration (Figure 6) occurred in the context of the overall taskinduced acceleration. By the current interpretation, this would indicate a brief period in which some capacity was held available for input. This seems reasonable given the subject's task of integrating new items with items in memory. ${ }^{4}$ Deceleration during learning was positively associated with rated efforts, suggesting that this deceleration was part of an active process and not simply a brief period of relaxation. The sensitivity of IBI to both capacity currently allocated and that held available provides a reasonable, although post hoc, basis for the divergence between probe RT and IBI for correct vs. error items. (In the absence of the dual sensitivity, IBI should be shortened when capacity, as indicated by RT, is greater for correct as compared to error items.)

\section{Changes Over Trials}

Experiment 2 examined learning over trials on the presumption that, as learning progressed, the processing capacity allocated to the learning task should decrease. Evidence of such a drop in capacity allocated occurred, but only in the initial lists of a session. Later lists showed a reversal, probably reflecting a general decrease in the alertness of the subjects. The IBI results for the experimental lists consistently showed a lengthening over trials. This would suggest a decrease in processing capacity allocated over trials, and correspond to the probe RT results for the initial lists. The IBI results for the rote lists may be interpreted to reflect the drop in alertness presumed to occur in experimental lists presented late in the session (cf. Lacey, 1972; Lawler, Obrist, \& Lawler, 1976). Additional confirming evidence is necessary, however, prior to interpretation of the joint changes in IBI and RT observed in lists presented late in a session. ${ }^{5}$

\section{Interpretative Summary}

Joint assessment of three measures potentially related to the allocation of processing capacity showed that changes in IBI were closely related to probe RT, but that effort ratings were related to neither RT nor IBI. Kahneman (1973) questioned the value of cardiac IBI for the assessment of processing capacity because of the bidirectionality of the response. The current approach suggests that this bidirectionality provides an assessment of the accessibility of processing capacity, while the degree of change provides an assessment of the amount of capacity allocated or held available. A close convergence of probe RT and IBI as measures of processing capacity allocated was possible when IBI was thus interpreted. Second-to-second results for IBI and RT showed no evidence that changes in capacity allocation were a function of momentary requirements of specific cognitive processes. Overall, the results suggest the promise of a psychophysiological approach to the study of processing capacity, although additional independent measures would be desirable. For example, a physiological measure independent of degree of change in IBI might be obtained by the recording of a sympathetic vascular index, skin conductance, pupillary responses, or cortical measures (see Harding, Stevens, \& Marston, 1973; Hillyard \& Picton, in press).

\section{REFERENCES}

BECKER, C. A. Allocation of attention during visual word recognition. Journal of Experimental Psychology: Human Perception and Performance, 1976, 2, 556-566.

BUtTer, M. J. Differential recall of paired associates as a function of arousal and concreteness-imagery levels. Journal of Experimental Psychology, 1970, 84, 252-256.

Coles, M. G. H., \& Duncan-Johnson, C. C. Cardiac activity and information processing: The effects of stimulus significance and detection and response requirements. Joumal of Experimental Psychology: Human Perception and Performance, 1975, 1, 418-428.

Craik, F. I. M., \& Blanksteln, K. R. Psychophysiology and human memory. In P. H. Venables \& M. J. Christie (Eds.), Research in psychophysiology. London: Wiley, 1975.

HAHN, W. W. Attention and heart rate: A critical appraisal of the hypothesis of Lacey and Lacey. Psychological Bulletin. 1973, 79, 59-70 
Harding, G. B., Stevens, E. E., \& Marston, P. T. Rate of information processing and skin resistance. Journal of Experimental Psychology, 1973, 99, 306-313.

Hillyard. S. A., \& Picton, T. W. Event-related brain potentials and selective information processing in man. In J. E. Desmedt (Ed.), Cerebral evoked potentials in man. London: Oxford University Press, in press.

Jennings, J. R. Cardiac reactions and different developmental levels of cognitive functioning. Psychophysiology, 1971, 8, 433-450.

JENNINGS, J. R. Information processing and concomitant heart rate changes in the overweight and underweight. Physiological Psychology, 1975, 3, 290-296.

Jennings, J. R., Averill, J. R., Opton, E. M., \& LAZARUS, R. S. Some parameters of heart rate change: Perceptual versus motor task requirements, noxiousness, and uncertainty. Psychophysiology, 1971, 7, 194-212.

Jennings, J. R., \& Wood, C. C. The $\varepsilon$-adjustment procedure for repeated-measures analyses of variance. Psychophysiology, 1976, 13, 277.278.

Jennings, J. R., \& Wood, C. C. Cardiac cycle time effects on performance, phasic cardiac responses, and their intercorrelation in choice reaction time. Psychophysiology, 1977, 14, 297-307.

Johnston, W. A., WagstafF, R. R., \& Griffith, D. Information-processing analysis of verbal learning. Journal of Experimental Psychology, 1972, 96, 307-314.

Kahneman, D. Attention and effort. Englewood Cliffs, N.J: Prentice-Hall, 1973.

Kahneman, D., Beatty, J., \& Pollack, I. Perceptual deficit during a mental task. Science, 1967, 157, 218-219.

Kantowitz, B. H., \& KNIGHT, J. L., JR. Testing tapping time-sharing. Journal of Experimental Psychology, 1974, $103,331-336$.

Kantowitz, B. H., \& KNIGet, J. L., JR. On experimenterlimited processes. Psychological Review, 1976, 83, 502.507.

KerR, B. Processing demands during mental operations. Memory \& Cognition, 1973, 1, 401.412.

KLEINSMITH. L. J., \& KAPLAN, S. Interaction of arousal and recall interval in nonsense syllable paired-associate learning. Journal of Experimental Psychology, 1964, 67, 124-126.

LABERGE, D. Acquisition of automatic processing in perceptual and associative learning. In P. M. A. Rabbit \& S. Dornic (Eds.), Attention and Performance V. London: Academic Press, 1975

LACEY, B. C., \& LACEY, J. I. Studies of heart rate and other bodily processes in sensorimotor behavior. In P. A. Obrist, A. Black, J. Brener, \& L. DiCara (Eds.), Cardiovascular psychophysiology-current issues in response mechanisms. biofeedback and methodology. Chicago: Aldine-Atherton, 1974.

LACEY, J. I. Some cardiovascular correlates of sensorimotor behavior: Example of visceral afferent feedback? In $\mathrm{C}$. $\mathrm{H}$. Hockman (Ed.), Limbic system mechanisms and autonomic function. Springfield, Ill: Thomas, 1972. Pp. 175-201.

LACEY, J. I., Kagan, J., LACEY, B. C., \& Moss, H. A. The visceral level: Situational determinants and behavioral correlates of autonomic response patterns. In P. H. Knapp (Ed.), Expression of the emotions in man. New York: International Universities Press, 1963. Pp. 161-196.

LAWLER, K. A., OBrust, P. A., \& LAwler, J. E. Cardiac somatic response patterns during a reaction time task in children and adults. Psychophysiology, 1976, 13, 448-455.

MostelleR, F., \& Rourke, R. E. K. Sturdy statistics: Nonparametrics and order statistics. Reading, Mass: Addison-Wesley, 1973.
Norman, D. A., \& Bobrow, D. G. On data-limited and resource-limited processes. Cognitive Psychology, 1975, 7 , 44-64.

Norman, D. A., \& Bobrow, D. G. On the analysis of performance operating characteristics. Psychological Review. 1976. 83, 508-510.

Posner, M. I. Psychobiology of attention. In C. Blakemore \& M. S. Gazzaniga (Eds.), The handbook of psychobiology. New York: Academic Press, 1974

Pribaum, K. H., \& McGuiness, D. Arousal, activation, and effort in the control of attention. Psychological Review. 1975, 82, 116-149.

Thorndike, E. L., \& LoRge, I. The teacher's word book of 30.000 words. New York: Columbia University Press, 1944.

Trumbo, D., \& Milone. F. Primary task performance as a function of encoding, retention, and recall in a secondary task. Joumal of Experimental Psychology, 1971, 91, 273-279.

Wallace, W. P. Review of the historical, empirical and theoretical status of the von Restorff phenomenon. Psychological Bulletin, 1965, 63, 410-424.

Walter, G. F., \& Porges, S. W. Heart rate and respiratory responses as a function of task difficulty: The use of discriminant analysis in the selection of psychologically sensitive physiological responses. Psychophysiology, 1976, 13. $563-571$.

WINER, B. J. Statistical principles in experimental design. New York: McGraw-Hill, 1971

\section{NOTES}

1. An analysis of probe RT and cardiac IBI from isolated item trials supported the absence of perceptual salience. The probe RT results showed the same relative values of isolated and nonisolated items as those in Figure 1; however, the differences were not as large. The cardiac IBI results were virtually identical between isolated and nonisolated items.

2. The results were also examined using the performance operating characteristic of Norman and Bobrow (1975). As the rewards for the primary and secondary tasks had not been overtly manipulated (see Norman \& Bobrow, 1976), the assumption was made that individuals would respond differently to the reward structure of the experiment, creating a situation where individual differences would reflect differences in resource allocation. Thus, the plot of probe RT vs. $P(C)$ anticipations was examined using the 12 subjects in the probe group. As would be expected, a linear relation was observed on the Trial 1 data that approached the vertical over trials as perfect serial anticipation was achieved. Similar results were obtained when a measure of cardiac acceleration was considered a measure of resource and plotted against $\mathrm{P}(\mathrm{C})$. In neither case, however, were the goodness of fit of the functions acceptable. Thus, this post hoc consideration of the results was not adequate to present arguments based on the performance operating characteristic concept.

3 . In addition to assessing group results, the performanceIBI relation may be assessed within each individual; that is, are trial-to-trial variations in IBI related to similar variations in correctness of anticipation? Within-subjects correlations of this type were computed; however, the direction and magnitude of the results were essentially the same as the group results reported.

4. It may be necessary to consider these relationships as applying only to relatively short-term memory. Butter (1970) has reported a recent replication of Kleinsmith and Kaplan's (1964) work suggesting that "arousal" related negatively to short-term memory and positively to long-term memory. 
The current approach rejects arousal as a unitary physiological concept and cumparison of results to this line of investigation is not fruitful given the physiological measures collected in the current study.

5. An editorial suggestion was made that the increase in RT over trials may reflect the increased load on memory over trials. Although such factors may ultimately have to be considered, little evidence of such a memory load effect was seen in the current experiments. First, such a memory load effect should be seen in all lists of an experimental day, not only in those run in the latter part of the session. Second, serial position analyses should reflect the increasing memory load during the final serial anticipation. No clear effects of this sort were observed.

(Received for publication July 22, 1977; accepted September 12, 1977.) 